Conclusion Our study suggests that loss of SMAD3 could possibly present a key event in the pathophysiology in PAH and restoring this pathway may provide therapeutic intervention.

Conflict of Interest The authors declare that there is no conflict of interest

\section{GETTING IT RIGHT FIRST TIME: CT CORONARY ANGIOGRAPHY FIRST IN GP PATIENTS REFERRED TO RACPC - IS IT FEASIBLE?}

John Graby, David Murphy, Rhys Metters, Katy Porter, Kady Parke, Eleanor Boden, Mzimkhulu Khumalo, Samantha Jones, Dawn Ellis, Rob Lowe, Jonathan Rodrigues. Royal United Hospitals Bath NHS Foundation Trust, Bath, UK

\subsection{6/heartjn-2021-BCS.184}

Introduction The Rapid Access Chest Pain Clinic (RACPC) targets rapid investigation, symptom relief and improved outcomes for patients with potential coronary artery disease (CAD). NICE recommends CT coronary angiography (CTCA) first line for all new onset chest pain patients without known CAD, unless pain is non-anginal with a normal ECG.

Getting It Right First Time reviews variations in service delivery to improve care, outcomes and efficiency. The RACPC pathway may be compared with 2 week wait cancer services, where investigation follows GP assessment prior to considering secondary care review.

In an era of assessing existing pathways to improve the patient journey, treatment and efficiency, this study aimed to test the hypothesis that an upfront CTCA strategy is feasible and safe for GP referrals to RACPC.

Methods A single-centre retrospective review of consecutive RACPC GP referrals September-October 2019, excluding known CAD or unavailable notes. An upfront CTCA following 'referral based triage' strategy (figure 1) was compared to existing face-to-face review and decision making. An analysis of referral content was made to facilitate safe central triage and cross-referenced with RACPC review and management. Time to diagnosis and hospital encounters were compared.

Results 107/172 patients met review criteria: median age 60 (IQR 22); 52 (49\%) male. GP Referral vs RACPC: Table 1 compares GP referral and RACPC findings. Of referrals without CAD history, no prior CAD was identified at RACPC review. All murmurs at referral were confirmed at RACPC. 17 (16\%) patients had murmurs newly identified at RACPC though none had $\geq$ moderate disease.

Actual management vs hypothetical pathway: 74 CTCAs were requested via RACPC vs 61 via a hypothetical pathway, the difference relating to requests outside of NICE guidance. This would extrapolate to a reduction of 78 CTCAs annually. Upfront CTCA cuts the time from referral to RACPC (median

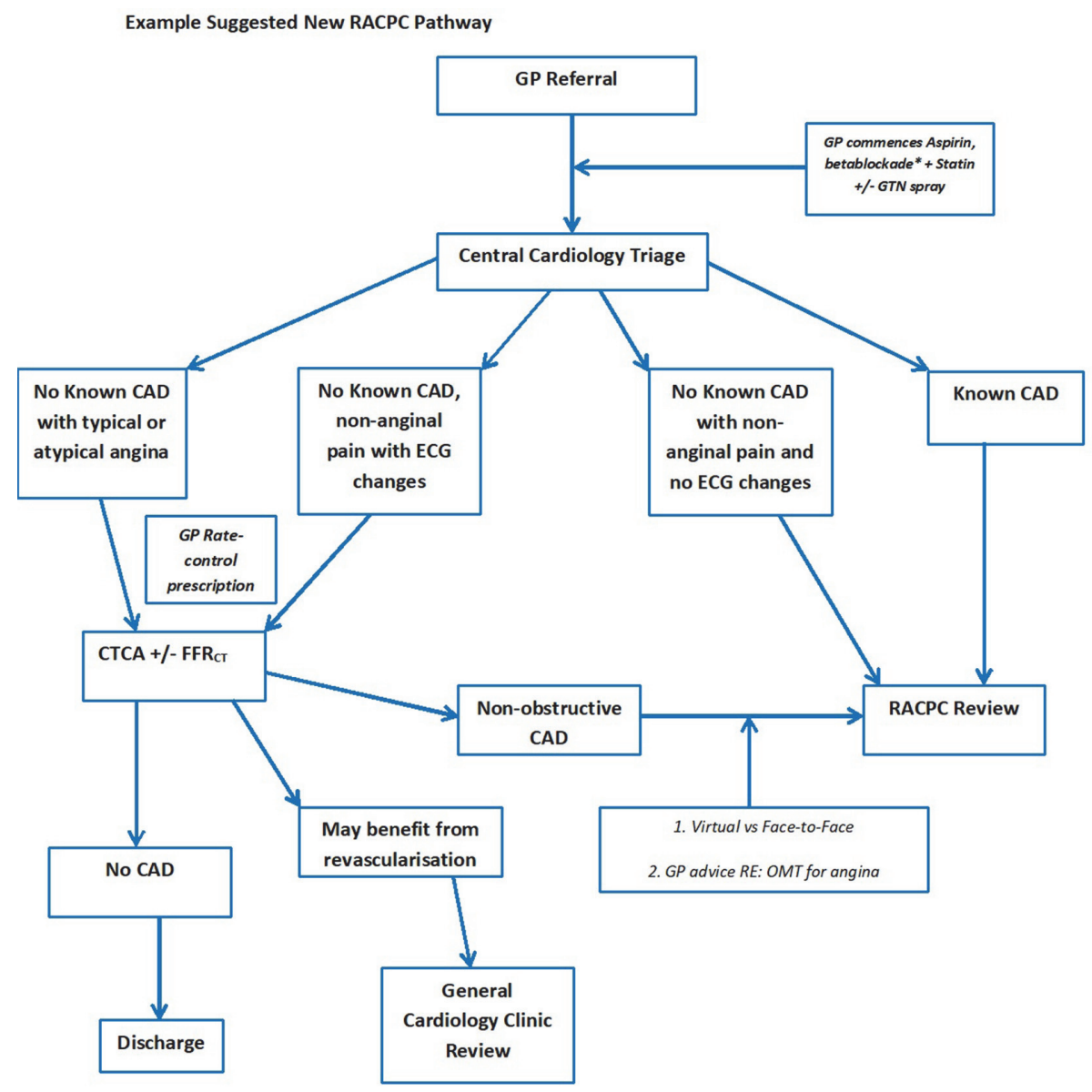

*If no contra indications or ivabradine 
Abstract 187 Table 1 Characteristics of GP referral documentation vs RACPC review

\begin{tabular}{|l|l|l|}
\hline Characteristic & GP Referral (\%) & RACPC (\%) \\
\hline Demographics & 100 & n/a \\
\hline CAD history documented & 93 & 100 \\
\hline Risk factors documented & 92 & 100 \\
\hline Presence/absence of murmur & 100 & 100 \\
commented on & & \\
\hline Chest pain category: & & \\
Typical & 35 & 24 \\
Atypical & 35 & 45 \\
Non-anginal & 30 & 25 \\
\hline ECG present & 100 & n/a \\
\hline
\end{tabular}

27 days [IQR 16.5]). Actual median time from referral to diagnosis combining all investigation pathways was 77 days (IQR 28), or 81 days (IQR 25) for obstructive CAD. This reduced by a median 22 days with the hypothetical pathway. 23 (21\%) patients with actual RACPC management had no CAD identified, representing pathways where 1 encounter (CTCA) may have been sufficient, which extrapolates to 138 less RACPC appointments annually $(£ 21,666$ in current NHS tariffs). $32(30 \%)$ patients had non-obstructive CAD and were discharged via letter.

Conclusion A novel pathway with a referral-based triage to upfront CTCA \pm subsequent RACPC would have been safe and efficient. The modelled reduction in hospital encounters would be cost effective and, importantly, enable the RACPC to review risk factors after identification of non-obstructive CAD. This improves treatment for a key group at risk of future events, particularly in those with aggressive CAD phenotypes on CTCA, whilst streamlining patients with obstructive CAD.

Conflict of Interest Nil

\section{IDENTIFICATION OF FAMILIAL HYPERCHOLESTEROLAEMIA IN PATIENTS PRESENTING WITH PREMATURE ACUTE CORONARY SYNDROME}

${ }^{1}$ James Duff*, ${ }^{2}$ Robin Wang, ${ }^{3}$ John Graby, ${ }^{4}$ Moya O'Doherty. 'North Bristol NHS Trust; ${ }^{2}$ Gloucestershire Hospitals NHS Trust, Gloucester, UK; ${ }^{3}$ Royal United Hospitals Bath NHS Foundation Trust; ${ }^{4}$ Royal United Hospitals, Bath

\subsection{6/heartjnl-2021-BCS.185}

Introduction Familial hypercholesterolaemia $(\mathrm{FH})$ is an underdiagnosed inherited disorder which confers substantial risk of premature cardiovascular disease. Over $90 \%$ of those with $\mathrm{FH}$ in the UK remain undiagnosed. Its importance was acknowledged in the 2019 NHS long term plan with an aim to identify $25 \%$ of $\mathrm{FH}$ patients by 2025. Patients with $\mathrm{FH}$ may present with an early acute coronary syndrome (ACS) which may represent the first opportunity for diagnosis. Identification of possible $\mathrm{FH}$ in this cohort enables specialist follow up, mitigation of risk and investigation of family members via cascade testing. We evaluated local disease burden, assessment of dyslipidaemia and identification of possible $\mathrm{FH}$ in a retrospective single centre review.

Methods A retrospective review was conducted of patients presenting with premature ACS to Royal United Hospitals Bath over a 1-year period.
Results We identified 96/573 (17\%) patients who presented with ACS under age 60. Although 77/96 (80\%) patients had cholesterol measured within 24 hours, only 13/96 (14\%) patients had a full lipid profile checked. 14/77 (18\%) had a total cholesterol $>6.0$, of whom $4 / 14$ (29\%), 4/14 (29\%) and $7 / 14(50 \%)$ had investigation for hypothyroidism, diabetes or alcohol excess respectively, as secondary causes for hypercholesterolaemia. $5 / 14(36 \%)$ patients with total cholesterol $>6.0$ had a family history taken with ages of relatives at onset of CVD; $4 / 5$ (80\%) of those asked had a family history of premature CVD. Upon review of high risk cases, 8 were deemed appropriate for referral to lipid clinic, while a further 5 patients may have warranted referral in the presence of a significant family history, but this information was absent in the medical notes. A decision to refer to lipid clinic was documented in 4 patients' notes, although only 2 referrals were received.

Conclusion We identified missed opportunities in the identification of genetic dyslipidaemia (including $\mathrm{FH}$ ) within a highrisk population. Further investigation through referral to lipid clinic was limited by a number of factors. Limited details of family history, particularly in patients with significant dyslipidaemia, is likely to contribute to under-identification of $\mathrm{FH}$ We identified variable risk stratification and reduced clarity on the accepted threshold for referral to lipid clinic as key barriers to appropriate diagnosis. We propose the introduction of a degree of protocolisation through an 'ACS proforma' and 'lipid clinic referral decision aid' to facilitate appropriate identification, assessment and management of patients with possible $\mathrm{FH}$.

Conflict of Interest None

\section{PREVALENCE OF COMORBIDITIES AND THE INCIDENCE OF CARDIOVASCULAR DISEASE IN THOSE WITH NON- CARDIAC CHEST PAIN}

${ }^{1}$ Sai Ramesh, ${ }^{1}$ Kayleigh Mason, 'James Bailey, ${ }^{2}$ Mamas Mamas, 'Kelvin Jordan. 'Keele University School of Medicine, UK; ${ }^{2}$ University of Keele

\subsection{6/heartjnl-2021-BCS.186}

Introduction Chest pain is a common presentation in primary care with many patients recorded with non-cardiac chest pain (NCCP), i.e. chest pain either unattributed to a cause or recorded with a non-cardiac reason. The relative prevalence of potential causes of NCCP in these patients and how these are related to risk of future cardiovascular disease (CVD) remains unclear. The aims of this study were to: (i) identify the 